\title{
A novel method of diagnosing premature ventricular contraction based on sparse auto- encoder and softmax regression
}

\author{
Jianli Yang, Yang Bai, Guojun Li, Ming Liu and Xiuling Liu* \\ Key Laboratory of Digital Medical Engineering of Hebei Province, College of Electronic and \\ Information Engineering, Hebei University, Baoding 071000, Hebei, China
}

\begin{abstract}
Premature ventricular contraction (PVC) is one of the most serious arrhythmias. Without early diagnosis and proper treatment, PVC can result in significant complications. In this paper, a novel feature extraction method based on a sparse auto-encoder (SAE) and softmax regression (SR) classifier was used to differentiate PVCs from other common NonPVC rhythms, including normal sinus (N), left bundle branch block (LBBB), right bundle branch block (RBBB), atrial premature contraction (APC), and paced beat (PB) rhythms. The proposed method was analyzed using 40 ECG records obtained from the MIT-BIH Arrhythmia Database. The proposed method exhibited an overall accuracy of $99.4 \%$, with a PVC recognition sensitivity and positive predictability of $97.9 \%$ and $91.8 \%$, respectively.
\end{abstract}

Keywords: PVC diagnosis, sparse auto-encoder, softmax regression, feature extraction

\section{Introduction}

The electrocardiogram (ECG), a bio-electric signal that records the electrical activities of the heart, is a noninvasive tool used to diagnose heart diseases [1]. Cardiac arrhythmia, defined as an irregular heartbeat, is a common cardiovascular disease [2]. In general, arrhythmias are recognized and classified based on ECG readings. Some common arrhythmias include left bundle branch block (LBBB), right bundle branch block (RBBB), premature ventricular contraction (PVC), atrial premature contraction (APC), and paced beat (PB). PVC, one of the most serious arrhythmias, is caused by the emission of a premature impulse from an ectopic pacemaker. PVCs are primarily characterized by ECG readings with broad, premature QRS complexes and no P waves. Recurrent PVCs can result in more serious complications, including angina, syncope, or heart failure [3]. Due to the uncertainty and randomness of PVCs, ECG readings must be dynamically observed over extended periods of time in order to accurately diagnose this type of arrhythmia. In addition, because of the large amounts of ECG data resulting from the rapid development of wearable devices, accurately and efficiently differentiating PVCs from other arrhythmias is difficult.

\footnotetext{
* Address for correspondence: Xiuling Liu, Key Laboratory of Digital Medical Engineering of Hebei Province, College of Electronic and Information Engineering, Hebei University, Baoding 071000, Hebei, China. Tel.: 0312-5079368; Fax: 03125079575; E-mail: liuxiuling121@hotmail.com.
} 
In recent years, many studies concerning the classification of arrhythmias have been conducted [412]. In one study, J. Wang developed a novel ECG arrhythmia classification method based on feature reduction by combing a principal component analysis (PCA) with a linear discriminant analysis (LDA). In addition, a probabilistic neural network (PNN) was used as a classifier to distinguish among eight different types of arrhythmias types [10]. In another study, M. Javadi extracted the morphological and time data from ECGs by combining mixture of experts (ME) and negatively correlated learning (NCL) in order to differentiate different types of arrhythmias [11]. L. Giovangrandi classified the ECG beats obtained from a large set of data using wavelet transforms and the timing information of a neural network classifier [12]. In [13], a PVC detection method based on a Discrete Wavelet Transform (DWT) was proposed. In the study, the wavelet coefficients of the ECG data were used as the feature vector, and a Support Vector Machine (SVM) was used as the classifier. In another study, a novel Bayesian classification system based on Gaussian process classifiers (GPC) was proposed for PVC detection [14]. The combination of GPC and an S-transform is advantageous compared to an SVM classifier in that the parameters of its kernel are automatically selected according to the Bayesian estimation procedure based on Laplace approximation. In [15], a novel PVC diagnostic method based on the chaotic features of ECGs was proposed. The results indicated that, using Lyapunov exponents, PVCs could be easily classified and differentiated from normal ECG beats and other arrhythmias. Peng Li developed a low complexity data-adaptive PVC recognition approach that exhibited good robustness against noise, generalization capabilities, and a PVC recognition accuracy of $98.2 \%$, indicating that it could be effectively used for real-time applications [16]. Using these algorithms, the features of ECGs were manually extracted based on time domain information, such as ECG morphology [6, 7, 11, 12], and transform domain information [4, 5, 9, 12-14], such as the wavelet transforms or statistical parameters $[10,15,16]$. These processes require artificial experience or specialized knowledge and increase computational complexity.

In recent years, deep learning has been successfully applied to various processes, such as dimensionality reduction, object recognition, and natural language processing [17]. In this paper, in order to extract more discriminational features from large amounts of ECG data using a neural network model and reduce the computational costs of diagnosing PVC, an automatic off-line PVC diagnostic method based on a deep learning model was proposed. The proposed method, a sparse autoencoder (SAE) and softmax regression (SR) classifier, was capable of efficiently differentiating between PVC and Non-PVC beats.

In Section 2, the feature extraction method based on the proposed sparse auto-encoder (SAE) and the softmax regression (SR) classifier is described in detail. The experimental results and discussion are presented in Section 3. The conclusions of this paper are presented in Section 4.

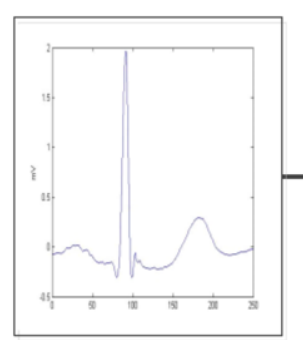

ECG beat

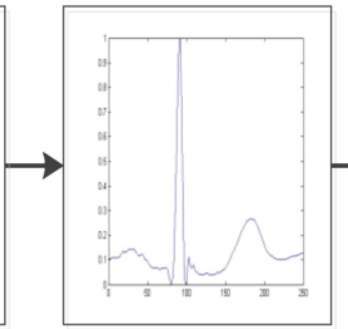

Normalization

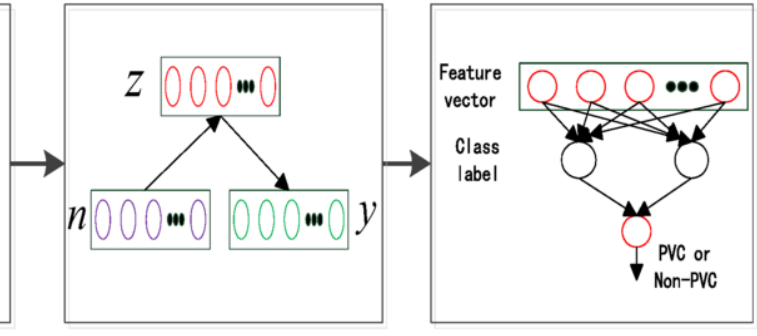

Feature extraction (SAE)

Classification (SF)

Fig. 1. Flow chart of the proposed method. 


\section{Methods}

In this paper, high-dimensional ECG beat data was normalized as the input vector of a sparse autoencoder (SAE). Next, SAE was used to extract the low-dimensional feature vector. A softmax regression (SR) was then used as a pattern classifier to differentiate between the PVC and Non-PVC beats via an extracted feature vector. A flow chart of the proposed method is shown in Figure 1.

\subsection{Feature extraction using the sparse auto-encoder}

An auto-encoder (AE) is a specialized neural network comprised of three layers, including a visible layer, hidden layer, and reconstruction layer. Auto-encoders are unsupervised learning algorithms that attempt to reconstruct visible layer data in the reconstruction layer [18]. In a sparse auto-encoder (SAE), a modified AE, the training method is constrained in order to give the hidden layer a sparseness property [19].

In this paper, the input vector of the SAE, which was defined as $x \in \mathfrak{R}^{v}$, was used to denote one $v$ dimensional ECG beat. The sigmoid function $f(a)=1 /(1+\exp (-a))$ was selected as the activation function of the SAE. Since the range of $f(\bullet)$ is $(0,1)$, the ECG beat $(x)$ was normalized to $n \in \mathfrak{R}^{v}$, with a range of $(0,1)$ The maximum and minimum amplitude values ( $x_{\max }$ and $x_{\min }$, respectively) of each ECG beat were used to normalize $x$ as

$$
n_{i}=\frac{x_{i}-x_{\min }}{x_{\max }-x_{\min }}
$$

where $i=1,2, \cdots, v$.

As illustrated in Figure 2, the SAE feature extraction process is comprised of two steps, in which (1) the encoder maps the normalized ECG beat $\left(n \in \mathfrak{R}^{v}\right)$ to activate the hidden layer $\left(z \in \mathfrak{R}^{h}\right)$, and (2) the decoder maps the activation $\left(z \in \mathfrak{R}^{h}\right)$ back to a reconstruction $\left(y \in \mathfrak{R}^{v}\right)$. These two steps can be expressed as

$$
z=f\left(W_{e} n+b_{e}\right)
$$

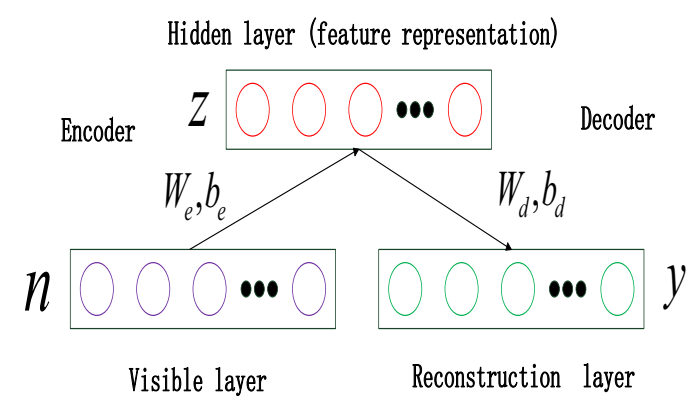

Fig. 2. Feature extraction with the sparse auto-encoder. 


$$
y=f\left(W_{d} z+b_{d}\right)
$$

where $W_{e} \in \mathfrak{R}^{h \times v}$ and $W_{d} \in \mathfrak{R}^{v \times h}$ are the weight matrices, and $b_{e} \in \mathfrak{R}^{h}$ and $b_{d} \in \mathfrak{R}^{v}$ are the bias vectors. The purpose of SAE training is to adapt the parameter set $\left\{W_{e}, W_{d}, b_{e}, b_{d}\right\}$ using backpropagation in order to minimize the cost function $(c(n, y))$, as shown by

$$
\underset{W_{e}, W_{d}, b_{e}, b_{d}}{\operatorname{argmin}}[c(n, y)]
$$

where $c(n, y)$ represents the "error" between the visible and reconstruction layer, which can be defined in a variety of ways. If the reconstruction layer data is capable of optimally reconstructing a normalized ECG beat, the activation of the hidden layer (denoted in red in Figure 2) can be regarded as an extracted feature representation of that normalized ECG beat. In this way, one $v$-dimensional ECG beat $(x)$ can be transformed into an $h$-dimensional feature vector $(z)$ and, thereby, the neutral network model can be used to extract the hidden discriminational features of that ECG beat.

The cost function $c(n, y)$ of the SAE can be defined as

$$
c(n, y)=\frac{1}{2 q} \sum_{i=1}^{q}\left\|n^{(i)}-y^{(i)}\right\|_{2}^{2}+\alpha \sum_{i=1}^{h} \mathrm{KL}\left(\rho \| \widehat{\rho}_{i}\right)
$$

where the first term is an average sum-of-squares error term with $q$ training samples of ECG beats, the second term is a sparse penalty term with $h$ neurons in the hidden layer, and $\alpha$ controls the weight of the sparse penalty term. The Kullback-Leibler (KL) divergence between two Bernoulli random variables in the sparse penalty term can be expressed as

$$
\mathrm{KL}\left(\rho \| \widehat{\rho}_{i}\right)=\rho \log \frac{\rho}{\hat{\rho}_{i}}+(1-\rho) \log \frac{1-\rho}{1-\widehat{\rho}_{i}}
$$

where $\widehat{\rho}_{i}=\frac{1}{q} \sum_{j=1}^{q} z_{i}\left(n^{(j)}\right)$ is the average activation of unit $i$ in the hidden layer, and $\rho$ is a sparsity target that must be selected.

\subsection{Classification using the softmax regression model}

The softmax regression (SR) model, which is extensively used during the supervised learning steps of deep neural networks, is a supervised learning algorithm. After extracting a feature vector, represented as the activation of the hidden layer in the SAE using ECG beats, the softmax regression (SR) model is used as a pattern classifier to differentiate between PVC and Non-PVC beats.

The SR model used in this paper is illustrated in Figure 3. In the model, $(z, l)$ is an SR training sample, in which $z \in \mathfrak{R}^{h}$ represents the extracted feature vector of an ECG beat, $l \in\{1,2\}$ represents 


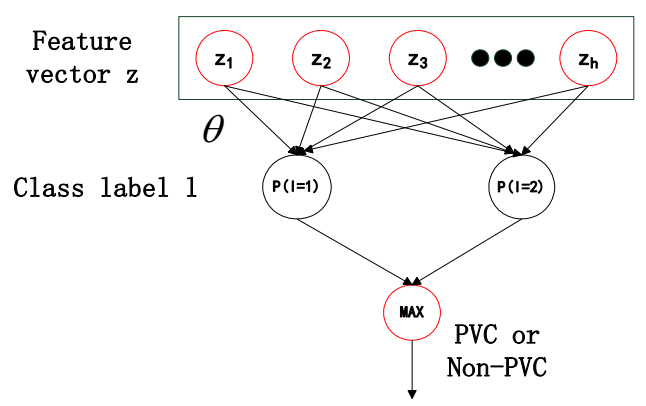

Fig. 3. Classification with the softmax regression model.

the class label, and the PVC and Non-PVC beats are denoted as ' 1 ' and ' 2 ', respectively. The hypothesis probability vector $\left(r_{\theta}(z)\right)$ of the softmax regression model used in this paper can be expressed as

$$
r_{\theta}(z)=\left[\begin{array}{l}
p(l=1) \\
p(l=2)
\end{array}\right]=\frac{1}{\sum_{i=1}^{2} e^{\theta_{i}^{T} z}}\left[\begin{array}{c}
e^{\theta_{1}^{T} z} \\
e^{\theta_{2}^{T} z}
\end{array}\right]
$$

where $\theta=\left[\begin{array}{ll}\theta_{1}^{T} & \theta_{2}^{T}\end{array}\right]^{T} \in \mathfrak{R}^{2 \times h}$ is the weight matrix, and $p(l=1), p(l=2)$ estimate the probability that a tested ECG beat is a PVC and Non-PVC, respectively. The class label with a higher probability is output as the classification result. In addition, $\sum_{i=1}^{k} e^{\theta_{i}^{T} h}$ normalizes the distribution. The cost function of the softmax regression model can be defined as

$$
J(\theta)=-\frac{1}{q}\left[\sum_{i=1}^{q} \sum_{j=1}^{2} 1\{l=j\} \log \frac{e^{\theta_{j}^{T} z}}{\sum_{m=1}^{2} e^{\theta_{m}^{T} z}}\right]
$$

where $1\{\cdot\}$ is the indicator function, such that 1 a true statement $\}=1$ and $1\{$ a false statement $\}=0$, and the model parameter $\theta$ was trained to minimize the cost function $(J(\theta))$

\section{Results and discussion}

\subsection{ECG beat sampling}

The ECG beats used in this paper were acquired from the MIT-BIH arrhythmia database, which is widely used as a standard database. In order to ensure the integrity of the data, 250 sampling points 

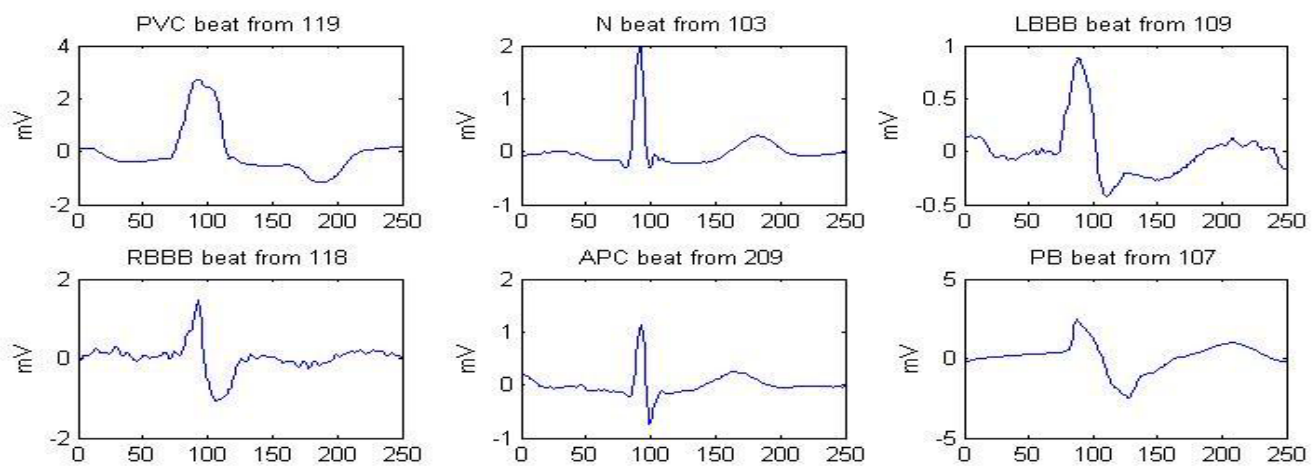

Fig. 4. ECG beat waveforms

were selected as single ECG beat samples. Of the samples, 89 were obtained from the left of the R peaks, and 160 were obtained from the right of the $\mathrm{R}$ peaks. The $\mathrm{R}$ peaks were obtained from the annotation files of the MIT-BIH arrhythmia database. For convenience, Non-PVC arrhythmias were defined as the arrhythmias including N, LBBB, RBBB, APC, and PB. The waveforms of the PVC and Non-PVC beats are displayed in Figure 4. The diagnostic performance of the proposed method was analyzed and tested using 80740 beats obtained from 40 ECG records from the MIT-BIH arrhythmia database. Of these randomly extracted beats, 4260 (obtained from files 106, 116, 119, 200, 201, 203, 210, 221, 223, and 233) were PVC beats, and 76480 (obtained from files 100, 101, 102, 103, 106, 107, $108,109,111,112,113,114,115,116,118,119,121,123,124,200,201,202,203,205,207,208$, $209,210,212,214,215,217,219,220,221,222,223,231,232$, and 233) were Non-PVC beats. Half of the beat data was used as the training set, and the other half was used as the testing set. The ECG beat selection method used in this study is shown in Table 1 .

Table 1

ECG beats analyzed in this paper

\begin{tabular}{|c|c|c|c|c|}
\hline Type & \multicolumn{2}{|c|}{ File } & Training set & Testing set \\
\hline \multirow[t]{10}{*}{ PVC } & \multicolumn{2}{|c|}{106} & 250 & 250 \\
\hline & \multicolumn{2}{|c|}{116} & 50 & 50 \\
\hline & \multicolumn{2}{|c|}{119} & 220 & 220 \\
\hline & \multicolumn{2}{|c|}{200} & 400 & 400 \\
\hline & \multicolumn{2}{|c|}{201} & 90 & 90 \\
\hline & \multicolumn{2}{|c|}{203} & 220 & 220 \\
\hline & \multicolumn{2}{|c|}{210} & 90 & 90 \\
\hline & \multicolumn{2}{|c|}{221} & 190 & 190 \\
\hline & \multicolumn{2}{|c|}{223} & 220 & 220 \\
\hline & \multicolumn{2}{|c|}{233} & 400 & 400 \\
\hline \multirow[t]{9}{*}{ Non-PVC } & \multirow[t]{9}{*}{$\mathrm{N}$} & 100 & 1100 & 1100 \\
\hline & & 101 & 800 & 800 \\
\hline & & 103 & 1000 & 1000 \\
\hline & & 106 & 700 & 700 \\
\hline & & 108 & 850 & 850 \\
\hline & & 112 & 1200 & 1200 \\
\hline & & 113 & 850 & 850 \\
\hline & & 114 & 900 & 900 \\
\hline & & 115 & 950 & 950 \\
\hline
\end{tabular}




\begin{tabular}{|c|c|c|c|c|}
\hline & & 116 & 1100 & 1100 \\
\hline & & 119 & 700 & 700 \\
\hline & & 121 & 900 & 900 \\
\hline & & 123 & 750 & 750 \\
\hline & & 200 & 800 & 800 \\
\hline & & 201 & 800 & 800 \\
\hline & & 202 & 1000 & 1000 \\
\hline & & 203 & 1200 & 1200 \\
\hline & & 205 & 1250 & 1250 \\
\hline & & 208 & 750 & 750 \\
\hline & & 209 & 1300 & 1300 \\
\hline & & 210 & 1200 & 1200 \\
\hline & & 215 & 1500 & 1500 \\
\hline & & 219 & 1000 & 1000 \\
\hline & & 220 & 950 & 950 \\
\hline & & 221 & 1000 & 1000 \\
\hline & & 222 & 1000 & 1000 \\
\hline & & 223 & 1000 & 1000 \\
\hline & & 233 & 1000 & 1000 \\
\hline & LBBB & 109 & 1200 & 1200 \\
\hline & & 111 & 1000 & 1000 \\
\hline & & 207 & 700 & 700 \\
\hline & & 214 & 1000 & 1000 \\
\hline & RBBB & 118 & 1000 & 1000 \\
\hline & & 124 & 700 & 700 \\
\hline & & 212 & 900 & 900 \\
\hline & & 231 & 600 & 600 \\
\hline & APC & 209 & 190 & 190 \\
\hline & & 222 & 100 & 100 \\
\hline & & 232 & 600 & 600 \\
\hline & PB & 102 & 1000 & 1000 \\
\hline & & 107 & 1000 & 1000 \\
\hline & & 217 & 700 & 700 \\
\hline Total & & & 40370 & 40370 \\
\hline
\end{tabular}

Table 2

Performance measurements

\begin{tabular}{|c|c|c|}
\hline \multicolumn{3}{|l|}{ Measurements } \\
\hline Total & PVC beats & Non-PVC beats \\
\hline$O A=\frac{N n+V v}{N n+N v+V n+V v} \times 100$ & $S e=\frac{V v}{V n+V v} \times 100$ & $S e=\frac{N n}{N n+N v} \times 100$ \\
\hline & $+P=\frac{V v}{N v+V v} \times 100$ & $+P=\frac{N n}{N n+V n} \times 100$ \\
\hline
\end{tabular}

Note: $O A$ : Overall accuracy (\%), Se: Sensitivity (\%), $+P$ : Positive predictability (\%), Nn (TN): Non-PVC beats recognized as Non-PVC beats (true negatives), $N v(F P)$ : Non-PVC beats recognized as PVC beats (false positives), $V \mathrm{n}(F N)$ : PVC beats recognized as Non-PVC beats (false negatives), $V v(T P)$ : PVC beats recognized as PVC beats (true positives).

\subsection{Performance evaluation}

A 250-20-250 sparse auto-encoder (SAE) was established in order to extract the 20-dimensional feature vector, which was defined as the activation of the hidden layer from the ECG beats in this 
paper. The sparsity target and weight of the sparse penalty term in Eq. (5) were defined as $\rho=0.2$ and $\alpha=3$, respectively, based on the results of numerous experiments.

The performance of the proposed method was evaluated using the statistical parameters shown in Table 2 .

Table 3

Diagnostic results

\begin{tabular}{|c|c|c|c|c|c|c|c|}
\hline \multirow[t]{3}{*}{ File } & \multicolumn{2}{|c|}{ Beats } & \multicolumn{5}{|c|}{ Measurements } \\
\hline & \multirow[t]{2}{*}{ PVC } & \multirow[t]{2}{*}{ Non-PVC } & \multirow[t]{2}{*}{$\mathrm{OA}$} & \multicolumn{2}{|l|}{ PVC } & \multicolumn{2}{|c|}{ Non-PVC } \\
\hline & & & & $\mathrm{Se}$ & $+\mathrm{P}$ & $\mathrm{Se}$ & $+\mathrm{P}$ \\
\hline 100 & 0 & 1100 & 100 & ------- & ------- & 100 & 100 \\
\hline 101 & 0 & 800 & 100 & ------- & ------- & 100 & 100 \\
\hline 102 & 0 & 1000 & 99.4 & ------- & 0 & 99.4 & 100 \\
\hline 103 & 0 & 1000 & 99.7 & & 0 & 99.7 & 100 \\
\hline 106 & 250 & 700 & 98.5 & 96.4 & 97.9 & 99.3 & 98.7 \\
\hline 107 & 0 & 1000 & 99.2 & ------- & 0 & 99.2 & 100 \\
\hline 108 & 0 & 850 & 99.3 & ------- & 0 & 99.3 & 100 \\
\hline 109 & 0 & 1200 & 98.9 & ------- & 0 & 98.9 & 100 \\
\hline 111 & 0 & 1000 & 99.7 & ------- & 0 & 99.7 & 100 \\
\hline 112 & 0 & 1200 & 99.8 & ------- & 0 & 99.8 & 100 \\
\hline 113 & 0 & 850 & 99.4 & ------- & 0 & 99.4 & 100 \\
\hline 114 & 0 & 900 & 100 & ------- & --- & 100 & 100 \\
\hline 115 & 0 & 950 & 99.4 & ------- & 0 & 99.4 & 100 \\
\hline 116 & 50 & 1100 & 99.3 & 94.0 & 90.3 & 99.5 & 99.7 \\
\hline 118 & 0 & 1000 & 99.3 & ------- & 0 & 99.3 & 100 \\
\hline 119 & 220 & 700 & 99.0 & 97.7 & 98.1 & 99.4 & 99.2 \\
\hline 121 & 0 & 900 & 100 & ------- & ------- & 100 & 100 \\
\hline 123 & 0 & 750 & 99.6 & ------- & 0 & 99.6 & 100 \\
\hline 124 & 0 & 700 & 99.1 & ------- & 0 & 99.1 & 100 \\
\hline 200 & 400 & 800 & 98.9 & 98.2 & 98.4 & 99.2 & 99.1 \\
\hline 201 & 90 & 800 & 98.8 & 94.4 & 94.4 & 99.3 & 99.3 \\
\hline 202 & 0 & 1000 & 100 & ------- & ------- & 100 & 100 \\
\hline 203 & 220 & 1200 & 99.2 & 95.9 & 99.0 & 99.8 & 99.2 \\
\hline 205 & 0 & 1250 & 99.6 & ------- & 0 & 99.6 & 100 \\
\hline 207 & 0 & 700 & 98.4 & ------- & 0 & 98.4 & 100 \\
\hline 208 & 0 & 750 & 100 & ------- & ------- & 100 & 100 \\
\hline 209 & 0 & 1490 & 99.6 & ------- & 0 & 99.6 & 100 \\
\hline 210 & 90 & 1200 & 99.5 & 93.3 & 100 & 100 & 99.5 \\
\hline 212 & 0 & 900 & 99.0 & ------- & 0 & 99.0 & 100 \\
\hline 214 & 0 & 1000 & 98.8 & -------- & 0 & 98.8 & 100 \\
\hline 215 & 0 & 1500 & 99.4 & ------- & 0 & 99.4 & 100 \\
\hline 217 & 0 & 700 & 99.1 & ------- & 0 & 99.1 & 100 \\
\hline 219 & 0 & 1000 & 100 & ------- & --- & 100 & 100 \\
\hline 220 & 0 & 950 & 100 & ------- & & 100 & 100 \\
\hline 221 & 190 & 1000 & 99.5 & 97.3 & 100 & 100 & 99.5 \\
\hline 222 & 0 & 1100 & 99.2 & ------- & 0 & 99.2 & 100 \\
\hline 223 & 220 & 1000 & 99.5 & 100 & 97.7 & 99.5 & 100 \\
\hline 231 & 0 & 600 & 98.8 & ------- & 0 & 98.8 & 100 \\
\hline 232 & 0 & 600 & 99.0 & ------- & 0 & 99.0 & 100 \\
\hline 233 & 400 & 1000 & 99.1 & 98.5 & 98.5 & 99.4 & 99.4 \\
\hline Total & 2130 & 38240 & 99.4 & 97.9 & 91.8 & 99.5 & 99.8 \\
\hline
\end{tabular}


Table 4

Diagnostic results

\begin{tabular}{llllll}
\hline Methods & \multicolumn{2}{l}{ Measures } & & \\
\hline & OA & PVC & \multicolumn{3}{l}{ Non-PVC } \\
\cline { 3 - 6 } & & Se & $+P$ & Se & $+P$ \\
\hline Manu [9] & 99.3 & 97.8 & 99.5 & ------- & -------- \\
Javadi [11] & 96.0 & 92.3 & ------ & 98.0 & ------- \\
Laurent [12] & 95.2 & 82.6 & 93.4 & ------ & ------ \\
Bazi [14] & 96.7 & 97.3 & 96.6 & ------ & ----- \\
Li [16] & 98.2 & 93.1 & 81.4 & 98.5 & 99.5 \\
Proposed method & 99.4 & 97.9 & 91.8 & 99.5 & 99.8 \\
\hline
\end{tabular}

Table 3 displays the experimental results. The proposed method exhibited an overall accuracy of 99.4\%, with a PVC recognition sensitivity and positive predictability of $97.9 \%$ and $91.8 \%$, respectively. In addition, the overall accuracy, PVC recognition sensitivity, and positive predictability of the proposed method were greater than $98.4 \%, 93.3 \%$, and $90.3 \%$, respectively, for all forty ECG readings. Thus, the proposed method was capable accurately detecting PVC beats.

In order to validate the proposed method, the results were compared to those of other methods. The results of the comparison are shown in Table 4. The overall accuracy, Non-PVC recognition sensitivity, Non-PVC positive predictability, and PVC recognition sensitivity of the proposed method were significantly higher than those of the other methods. However, the proposed method exhibited poorer PVC positive predictability than the other methods. This could be because the PVC beats were only extracted from files 106, 116, 119, 200, 201, 203, 210, 221, 223, and 233. These ten files had higher rates of ventricular premature beats. Since no PVC beats were extracted from the remaining file, the PVC positive predictability of the remaining files was equal to 0 or did not exist, decreasing the overall PVC positive predictability. If only these files were included, a PVC positive predictability of $98.2 \%$ could be achieved.

\section{Conclusions}

In this paper, a novel feature extraction method based on a sparse auto-encoder (SAE) and softmax regression (SR) classifier was proposed for the diagnosis of PVC. In the proposed method, a neural network model, sparse auto-encoder (SAE), was used to extract the discriminational features in large amounts of ECG data without the need for manual feature vector design or selection, reducing the computational costs and improving the accuracy of PVC diagnostic tasks. PVCs were easily differentiated from common Non-PVC beats using the extracted feature through the softmax regression (SR) classifier. A total of 80740 beats obtained from 40 ECG records from the MIT-BIH arrhythmia database were used to validate the method. The experimental results indicated that the proposed method exhibited an overall accuracy of $99.4 \%$ and a PVC recognition sensitivity of $97.9 \%$. In the future, the influence of the sparse auto-encoder (SAE) model parameters on the classification results will be studied, and deep neutral network stacking multi-layer auto-encoders will be constructed in order to develop automatic diagnostic tools for other types of arrhythmias.

\section{Acknowledgments}


This work is supported by projects of the National Natural Science Foundation of China (61473112 and 61203160) and the Natural Science Foundation of Hebei Province (F2015201112).

\section{References}

[1] M. Korurek and B. Dogan, ECG beat classification using particle swarm optimization and radial basis function neural network, Expert Systems with Applications 37 (2010), 7563-7569.

[2] E. Sandoe and B. Sigurd, Arrhythmia: A Guide to Clinical Electrocardiology, Publishing Partners, Bingen, 1991.

[3] K. Sawasaki and M. Saito, A case of heart failure caused by frequent premature ventricular contractions, Journal of Arrhythmia 26 (2010), 204-208.

[4] S.X. Yang, C.M. Chen, Y.L. Dai and G.Y. Yang, The research of arrhythmia algorithm based on fuzzy neural network, Third International Symposium on Electronic Commerce and Security (ISECS), Guangzhou, China, 2010, pp.131-133.

[5] D.F Ge, Study of ECG feature extraction for automatic classification based on wavelet transform, The 7th International Computer Science \& Education (ICCSE), Melbourne, Australia, July 14-17, 2012, pp. 500-503.

[6] P. de Chazal, M. O'Dwyer and R.B. Reilly, Automatic classification of heartbeats using ECG morphology and heartbeat interval features, IEEE Transactions on Biomedical Engineering 51 (2004), 1196-1206.

[7] Z. Zidelmal, A. Amirou, D. Ould-Abdeslam and J. Merckle, ECG beat classification using a cost sensitive classifier, Computer Method and Programs in Biomedicine 3 (2013), 570-577.

[8] P. Melin, J. Amezcua, F. Valdez and O. Castillo, A new neural network model based on the LVQ algorithm for multiclass classification of arrhythmias, Information Science 279 (2014), 483-479.

[9] Manu Thomas, Manab Kr Das and Samit Ari, Automatic ECG arrhythmia classification using dual tree complex wavelet based features, International Journal of Electronics and Communications 69 (2015), 715-721.

[10] J. Wang, W. Chiang, Y. Hsu and Y.C. Yang, ECG arrhythmia classification using a probabilistic neural network with a feature reduction method, Neurocomputing 116 (2013), 38-45.

[11] M. Javadi, Seyed Ali Asghar Abbaszadeh Arani, A. Sajedin and R. Ebrahimpour, Classification of ECG arrhythmia by a modular neural network based on mixture of experts and negatively correlated learning, Biomedical Signal Processing and Control 8 (2013), 289-296.

[12] Laurent Giowangrandi and G.T.A. Kovacs, Robust neural network based classification of premature ventricular contractions using wavelet transform and timing interval features, IEEE Transactions on Biomedical Engineering $\mathbf{5 3}$ (2006), 2507-2515.

[13] A. Orozco-Duque and F.J. Martinez-Tabares, Classification of premature ventricular contraction based on discrete wavelet transform for real time applications, Pan American Health Care Exchanges, Medellin, 2013, pp. 1-5.

[14] Y. Bazi and H. Hichri, Premature ventricular contraction arrhythmia detection and classification with Gaussian process and $\mathrm{S}$ transform, Fifth International Conference on Computational Intelligence, Communication Systems and Networks, Madrid, June 5-7, 2013, pp. 36-41.

[15] Haiman Du, Yang Bai, Z. Zhou and H. Wang, A novel method for diagnosing premature ventricular contraction beat based on Chaos theory, The 11th International Conference on Fuzzy Systems and Knowledge Discovery, Xiamen, China, Aug. 19-21, 2014, pp. 497-501.

[16] Peng Li, Chengyu Liu, Xinpei Wang, Dingchang Zheng, Yuanyang Li and Changchun Liu, A low-complexity dataadaptive approach for premature ventricular contraction recognition, Signal, Image and Video Processing 8 (2014), 111120 .

[17] Y. Bengio, Learning deep architectures for AI, Foundations and trends in Machine Learning 2 (2009), 1-127.

[18] Y. Bengio, Lamblin, P. Popovici and D. Larochelle, Greedy layer-wise training of deep networks, Advances in Neural Information Processing Systems 19 (2007), 153-160.

[19] L. Goodfellow, H. Lee, Q.V. Le, Andrew Saxe and Andrew Y. Ng, Measuring invariances in deep networks, Advances in Neural Information Processing Systems 22 (2009), 646-654. 